\title{
El metablog OpenMethods o cómo abrir la investigación en Humanidades Digitales
}

\author{
Gimena del RIo RIANDE \\ Instituto de Investigaciones Bibliográficas y Crí- \\ tica Textual, IIBICRIT-CONICET, Argentina \\ gdelrio@conicet.gov.ar \\ https://orcid.org/0000-0002-8997-5415
}

\author{
ERZsÉBET TÓTH-CZIFRA \\ Center Marc Bloch - DARIAH -UE, Berlin, Alemania \\ erzsebet.toth-czifra@dariah.eu \\ https://orcid.org/0000-0002-5350-067X
}

\section{OpenMethods Metablog or How to Open Digital Humanities Research}

\begin{abstract}
RESUMEN
¿De qué modo pueden las Humanidades Digitales colaborar con los procesos de apertura en la Academia, no solo en términos de acceso, sino también en lo que hace a métodos, mejores prácticas y oportunidades de colaboración? ¿Y qué aspecto debe tener esta apertura en el contexto del movimiento de ciencia abierta a escala global? Este artículo se propone reflexionar sobre las posibilidades que ofrece el proyecto OpenMethods para el desarrollo de unas humanidades digitales más abiertas, no solo en términos de colaboración o democratización del conocimiento a través de recursos y tecnologías abiertas, sino en tanto herramienta que ayudaría a promover y estandarizar elementos como el descubrimiento, la interoperabilidad, la accesibilidad, la reproducibilidad (los principios FAIR) y el reuso en el ámbito de investigación de las Ciencias Humanas y Sociales.
\end{abstract}

PALABRAS CLAVE Humanidades digitales; Metablog; Recursos de investigación abiertos; Colaboración; Reúso

\section{ABSTRACT}

How can Digital Humanities collaborate with open processes in Academia, not only in terms of access, but also in terms of methods, best practices and opportunities for collaboration? And what should this opening look like in the context of the global open science movement? This article aims to reflect on the possibilities offered by the OpenMethods project for the development of open Digital Humanities, not only in terms of collaboration or knowledge democratization through open resources, data and technologies, but also promoting and standardizing elements such as findability, accessibility, interoperability, reproducibility (FAIR principles) and reuse in the Humanities and Social Sciences research. 


\section{Humanidades Digitales ¿abiertas?}

Con la publicación del primer Companion to Digital Humanities (Schreibman et al., 2004) las prácticas hasta entonces conocidas como Humanities Computing -en español, Informática Humanística- se rebautizaron en lo que hoy llamamos Digital Humanities. Los editores del Companion... entendieron que la evolución de los primeros trabajos de aplicación de herramientas y recursos electrónicos en proyectos más complejos, desde lo disciplinario y lo tecnológico, junto con la incorporación de marcos teóricos provenientes de las Ciencias Sociales y la Informática, constituían un nuevo campo. A pesar de que el origen del término es disputado', y que hoy contamos con una variedad de iniciativas a nivel global, es aproximadamente desde ese entonces que a los proyectos de investigación en Humanidades que proponen un trabajo desde la aplicación de metodologías y herramientas computacionales -cuantitativas o cualitativas-, se los enmarca en el campo de las Digital Humanities o, en español, Humanidades Digitales.

Las Digital Humanities tienen un largo recorrido en las academias norteamericanas y europeas, desde el señero proyecto del jesuita Roberto Busa con IBM -el Index Thomisticus-, comenzado en la década del 50 del siglo XX (Vanhoutte, 2013; Jones, 2016) y se construyen hoy allí no solo a través de proyectos de investigación, sino también en carreras de posgrado, cursos de verano, centros, laboratorios, sitios web, blogs, revistas científicas y asociaciones que legitiman su discurso y sus prácticas (Kirschenbaum, 2010; del Rio Riande, 2015 y 2016). En los últimos años, principalmente en Europa, infraestructuras como DARIAH o Clarin han trabajado en el fortalecimiento de plataformas, herramientas y metodologías digitales para el trabajo de investigación en Humanidades.

Más allá de los abordajes particulares, y de las definiciones más o menos disciplinarias (por ejemplo, Sahle, 2013; Galina, 2014), en la mayor parte del Norte Global las Humanidades Digitales crecen a caballo entre las Humanidades, y las Ciencias de la Información y los métodos, lenguajes y programas informáticos, privilegiando los análisis de grandes corpus, la minería y visualización de datos, la edición digital y la construcción de bibliotecas digitales, o la reflexión crítica sobre herramientas, métodos y metodologías. Sin embargo, por citar un ejemplo, en Latinoamérica la situación es algo diferente, y las Humanidades Digitales latinoamericanas constituyen aún un campo emergente, mucho más relacionado con tradiciones epistemológicas más ancladas en la región, como los estudios sobre Medios o los de Ciencia, Tecnología y Sociedad (CTS), o pensadas desde sus posibilidades en la democratización del conocimiento a través de la web (Fiormonte y del Rio Riande, en prensa). Así mismo, en la mayoría de los países de esa región, las Humanidades Digitales son aún un espacio sin financiación o infraestructuras específicas (del Rio Riande, 2015) que interpela las barreras tecnológicas que supone ese tipo de investigación, hecho sobre el que pocas investigacio- nes han puesto sus ojos (Fiormonte, 2012; 2014).

Sorprendentemente, los debates sobre la apertura de los datos o cuestiones como la reproducibilidad de un proyecto de investigación y de sus métodos, así como la de lo abierto -conceptos en estrecha relación ${ }^{2}$ - no han sido un sitio de reflexión demasiado visitado en las Humanidades Digitales del Norte o del Sur. Por citar una vez más el Companion..., este apenas menciona el término open en unas 93 ocasiones, y solo en los capítulos sobre edición digital académica o bibliotecas digitales lo relaciona con términos como software o archive (del Rio Riande, 2018b). Y tampoco las Best Practices in Digital Humanities (Nebraska, New York University, Stanford, entre otras) o las propuestas de evaluación de la RedHD o la Sociedad Humanidades Digitales Hispánicas ${ }^{3}$, entre muchas otras, han hecho foco en los beneficios de lo abierto en la elección de software, hardware, reproducibilidad, o métodos de trabajo y colaboración en un proyecto de investigación en Humanidades Digitales. Si bien es verdad que muchos proyectos que hacen hincapié en acceso abierto, formatos de publicación, evaluación académica y plataformas digitales han surgido en el seno de las Humanidades y las bibliotecas -Hirmeos, Knowledge Unlatched, Language Science Press, Linguistics in Open Access, Open Edition, u Open Library of Humanities, o los metapublishers Scielo y Redalyc (Tóth-Czifra, 2019)-, hasta el momento, muy pocas iniciativas dedicadas a las Humanidades Digitales -entre las que destacamos Open Context, Perseus Digital Library, Collections as Data, o el Digital Humanities Data Curation Institute- han reflexionado sobre las ventajas de lo abierto, no solo en términos de datos, recursos y presupuestos, sino en tanto elemento estándar que promueve elementos como la interoperabilidad, la reproducibilidad y el reuso ${ }^{4}$.

En nuestro caso, con una aproximación bilingüe (inglés-español) y global, buscamos con Open in $\mathrm{DH}$ (del Rio Riande et al., 2018; Schallier et al., 2018) ${ }^{5}$ desde 2016 acercarnos a unas Humanidades Digitales más abiertas e inclusivas. Desde un lugar relacionado con sus métodos y metodologías, propusimos, en un principio, el proyecto Metodologías de Investigación en Herramientas Digitales para la Investigación en Humanidades y Cs. Sociales (MHeDI), que dio origen al actual Laboratorio de Humanidades Digitales (HD CAICYT Lab). En la misma estela, a través del proyecto OpenMethods estamos trabajando, junto a una amplia comunidad de investigadores en publicaciones digitales sobre métodos digitales para la investigación en Humanidades Digitales disponibles en abierto, apelando al multiligüismo, a la diversidad, y a la selección y curaduría colectivas.

\section{Lo abierto y lo digital: posibilidades a explorar por las Humanidades Digitales}

Aunque las políticas de acceso abierto varían de país a país, la visibilidad y la difusión de la investigación y su impacto, y la inserción de la investigación en el ámbito de la educación 


\section{The old way of doing research}

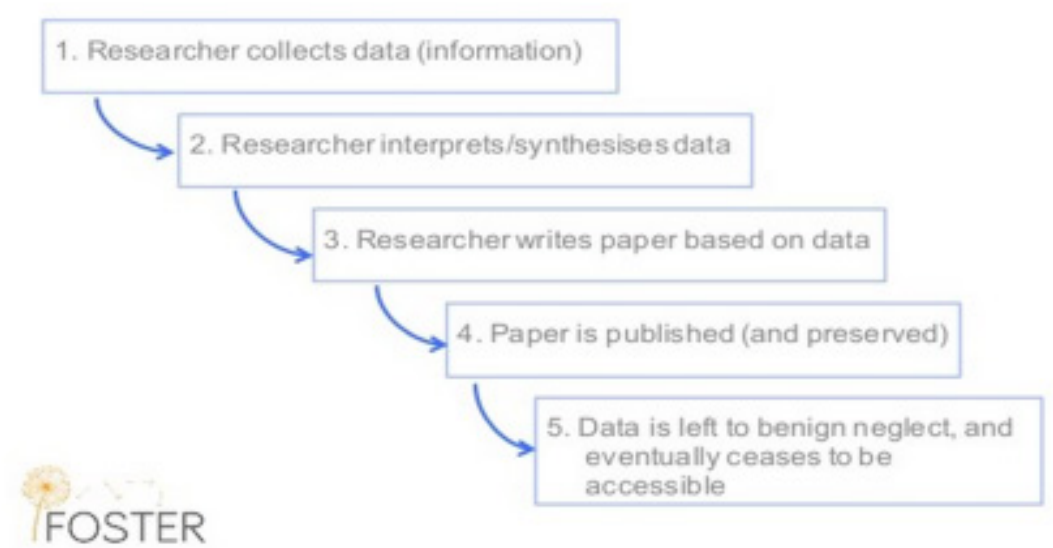

Figura 1. El viejo modo de hacer investigación. Fuente: FOSTER

y en la sociedad, a través de las posibilidades de difusión masiva de internet, son algunos objetivos compartidos. De acuerdo con las diferentes declaraciones del movimiento de Acceso Abierto, la equidad y la inclusión a escala global se encuentran entre sus preocupaciones centrales, aunque no exista un único criterio acerca de cómo alcanzar estos objetivos y, dependiendo de las regiones, las perspectivas puedan ser muy diferentes ${ }^{6}$. Por ejemplo, en los Estados Unidos y Europa los editores comerciales han desempeñado durante mucho tiempo un papel central en la publicación periódica, sin embargo, en otras partes del mundo, como América Latina, la publicación comercial no está tan extendida, ya que la investigación ha estado financiada casi exclusivamente con dinero público (Appel et al., 2018) y, de este modo, los modelos alternativos crecen con apoyo de los gobiernos y de la comunidad científica en general y el conocimiento producido en la academia se entiende como un bien común (Hess y Ostrom, 2016).

En los últimos tiempos, nuevas aproximaciones como las de la Investigación Abierta o Ciencia Abierta surgieron como definiciones más abarcativas que buscan introducir nuevas agendas en el movimiento del Acceso Abierto, redefiniendo las características de la publicación científica y considerando la producción completa de conocimiento como un ciclo en el que lo abierto se mantiene desde el origen hasta el resultado. La Ciencia Abierta aboga por una mayor transparencia en los métodos, la reutilización, participación, cooperación y reproducibilidad en la investigación, en el uso de software e infraestructuras abiertos, y en el acceso a publicaciones, recursos y datos científicos, así como en la evaluación científica:

Open Science is the practice of science in such a way that others can collaborate and contribute, where research data, lab notes and other research processes are freely available, under terms that enable reuse, redistribution and reproduction of the research and its underlying data and methods (FOSTER).

El core de la investigación en Humanidades Digitales son los proyectos que se expresan a través de repositorios y bases de datos en línea, ediciones digitales académicas, blogs, o bibliotecas digitales disponibles, en su amplísima mayoría, de forma gratuita y online. La investigación abierta parece haber nacido con las Digital Humanities, aunque poco se haya relacionado hasta ahora con ella. Será tal vez que, como bien señala Kathleen Fitzpatrick (2010):

(...) the key problems that we face again and again are social rather than technological in nature: problems of encouraging participation in collaborative and collective projects, of developing sound preservation and sustainability practices, of inciting institutional change, of promoting new ways of thinking about how academic work might be done in the coming years.

Fitzpatrick hace hincapié en la necesidad de un cambio de mentalidad dentro de las instituciones, tanto en el apoyo a las nuevas formas de producción de conocimiento, a través de la publicación en medios digitales, la evaluación de lo inter y transdisciplinar, lo abierto, lo colectivo y lo cooperativo, y a procesos y resultados que hacen tanto a la escala de lo cualitativo como con la de lo cuantitativo. Lo aportado por esta investigadora bien se relaciona con las propuestas actuales de la Ciencia Abierta y los procesos de apertura que buscan superar el viejo modo de hacer investigación, que privilegia la investigación individual, la publicación en revistas de impacto y no accesibilidad a los datos, como objeto que pertenece únicamente al investigador y su investigación (ver figura 1).

En nuestra opinión, las Humanidades Digitales pueden potenciar la sinergia entre lo abierto y lo digital. Una edición digital, 


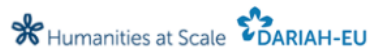 \\ OPENMETHODS}

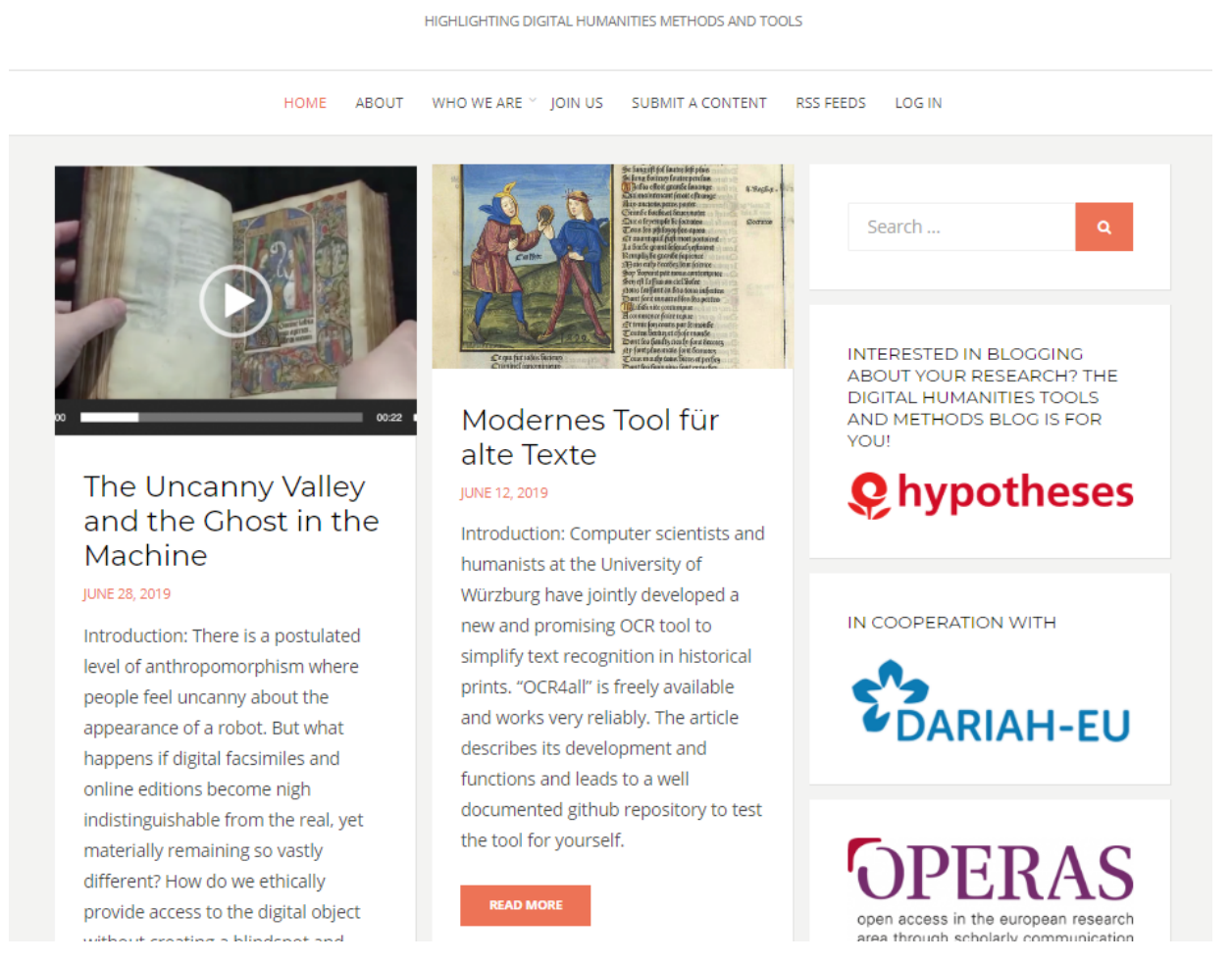

Figura 2. Interfaz del metablog OpenMethods.

un proyecto de mapeo o visualización distante (Moretti, 2005) busca, al igual que el Acceso Abierto, eliminar las barreras inherentes a la cultura de la impresión, como las limitaciones en el alcance y la distribución, las restricciones en los números de página y las dificultades para vincular los materiales de origen. Al hacer hincapié en los datos como elemento vertebrador, las Humanidades Digitales nos permiten también reflexionar sobre nuestros procesos de investigación. Este es el lugar desde donde se construye epistemológicamente el proyecto OpenMethods.

\section{OpenMethods: abrir la investigación en Humanidades Digitales}

\subsection{Objetivos del proyecto OpenMethods}

Una de las principales barreras para establecer formas más abiertas de producción y difusión de conocimiento radica probablemente en las formas de comunicación académica que privilegian las prácticas de investigación individual y la publicación científica relacionada con productos y no con procesos. En el campo de las Humanidades Digitales, el acceso a datos o a las características del software utilizado resulta esencial para una mejor comprensión de los proyectos. Así, los investigadores tienen la necesidad de complementar las prácticas tradicionales de publicación, como libros y artículos de revistas, con otras prácticas de comunicación académica que faciliten la disponibilidad inmediata en línea de resultados o procesos de investigación. El metablog OpenMethods tiene como objetivo explorar y ofrecer una solución para esta necesidad y avanzar así en el establecimiento de una cultura de investigación abierta dentro de las Humanidades Digitales (figura 2).

En lo que sigue describiremos cómo buscamos acercarnos a este objetivo: proporcionaremos una descripción general del proyecto y cómo se organiza nuestro flujo de trabajo para servir a una curación de contenido eficiente y dirigida por la comunidad, contribuyendo a establecer una cultura de investigación abierta en nuestro campo. Concluiremos con una breve discusión sobre los desafíos del proyecto en lo que hace a la participación a nivel global, la diversidad y el multilingüismo.

\subsection{Cómo el flujo de trabajo y la infraestructura de OpenMethods potencian la colaboración a través del reuso y la curaduría de contenido}

Si el Acceso Abierto posibilita, a través de la web, la consulta y el reuso de los recursos derivados de la producción científica o académica, tales como artículos publicados en revistas de acceso abierto, imágenes, documentos audiovisuales, etc., sin restricciones económicas (Suber, 2006), el metablog OpenMethods sostiene estos principios, proporcionando una 
plataforma que busca reunir, a través de distintos elementos tecnológicos, publicaciones digitales de distinto género y formato, y en distintas lenguas, sobre métodos de las Humanidades Digitales disponibles en abierto, apelando a la agilidad de la plataforma (Anderson y McPherson, 2011) y, al mismo tiempo, a la construcción colaborativa del conocimiento. Los temas de interés de OpenMethods son descripciones de métodos y herramientas, herramientas y métodos críticos, así como reflexiones prácticas y teóricas sobre cómo y por qué la investigación en humanidades se realiza digitalmente y cómo la creciente influencia de los métodos y herramientas digitales cambia las actitudes académicas y las prácticas científicas de la investigación en Humanidades.

Teniendo en cuenta que son siempre los investigadores quienes mejor pueden evaluar qué investigación es relevante para ellos y sus comunidades (Tennant, 2016), OpenMethods se propone como un espacio web abierto y colaborativo donde los expertos de todo el mundo -desde jóvenes investigadores, estudiantes o expertos de renombre- pueden recomendar, seleccionar, nominar y/o curar contenido publicado en línea y en abierto sobre distintos métodos en Humanidades Digitales (estándares de edición, explotación de corpus, visualización de datos, etc.) en cualquier lengua. Lo interesante es que el contenido que la plataforma busca aunar se basa en el reuso, ya que republica trabajos previamente publicados en distintos formatos y lenguas en abierto, ofreciendo una mayor visibilidad y reconocimiento de los resultados de investigación. En ese sentido, su trabajo se encuentra en la misma línea que los metapublishers de artículos académicos en América Latina, SCIELO y RedAlyC y hasta, nos animaríamos a decir, en el concepto de acceso abierto sin fines comerciales, y la defensa del conocimiento como un bien público que busca la circulación de sus contenidos en plataformas que permiten el acceso y uso de estos (Alperin y Fischman, 2015).

OpenMethods es intencionalmente un espacio de reuso y multilingüismo que busca facilitar el debate inter pares y la difusión del conocimiento sobre Humanidades Digitales. A pesar de que el proyecto se inició en el año 2017 desde la infraestructura pan-europea DARIAH, como heredero del proyecto Humanities at Scale (Engelhardt et al., 2017), es hoy día gobernado por grupo de expertos en Humanidades Digitales de distintos países: específicamente, 27 editores de 12 países? En nuestra opinión, la apertura del acceso al conocimiento académico también debería incluir una representación más equitativa de la producción de conocimiento en todo el mundo. Por ello, nuestro equipo editorial selecciona contenido en 16 idiomas: croata, checo, holandés, inglés, francés, alemán, griego, húngaro, italiano, polaco, portugués, ruso, serbio, eslovaco, español y sueco. El inglés es solo comprendido como la lengua que es compartida por la mayor parte de la comunidad y que sirve para glosar contenido 8 .

Es interesante destacar que, como decíamos más arriba, el proceso comprendido entre la nominación de republicación de contenido en acceso abierto no se limita a las sugerencias de nuestro Equipo Editorial, sino que está abierto a cualquier experto que, de forma anónima o por nombre a través de un navegador o la etiqueta de Twitter @openmethods_dh, desee proponer un texto sobre métodos en las Humanidades Digitales en cualquier lengua. La editora principal, Erzsébet Tóth-Czifra revisa el contenido propuesto y lo discute desde el interior de la plataforma con el Equipo Editorial, que es quien, finalmente, toma la decisión sobre qué contenido debe ser republicado en el metablog.

Tecnológicamente, la plataforma de OpenMethods, a pesar de que no está basada en software abierto, está preparada para ser lo más abierta posible, en el sentido de tecnología simple e intuitiva, al utilizar uno de los CMS más simples y extendidos para en trabajo web, WordPress. Desde nuestro punto de vista, el poder de OpenMethods reside en las personas que se acercan de uno u otro modo a la plataforma, no en un avance tecnológico No obstante, los desarrollos realizados para este proyecto y los complementos que usamos son de código

\section{Integration with ISIDORE and NERD}

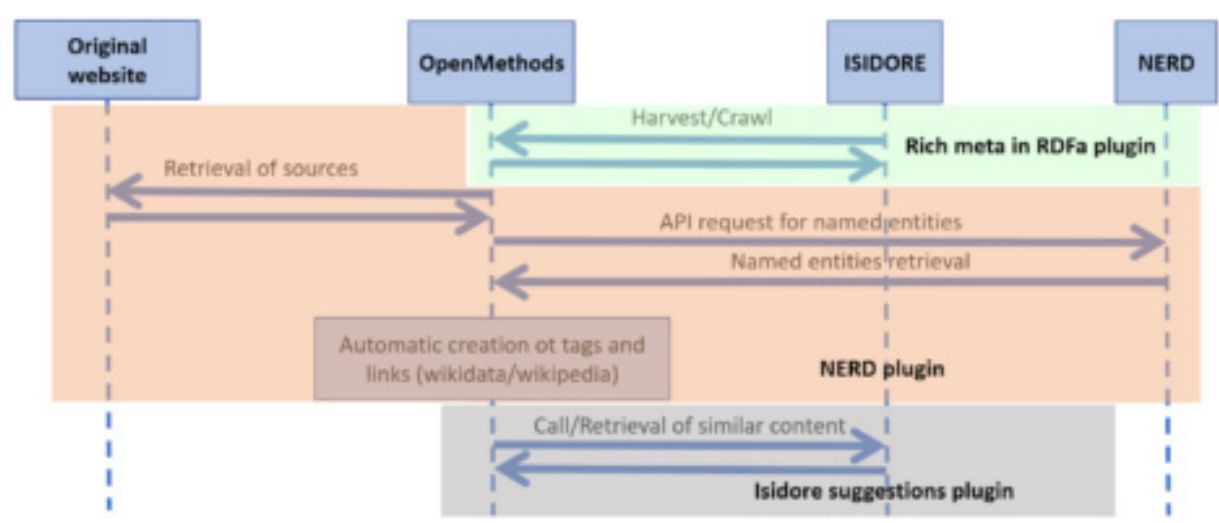

Figura 3. Integración de OpenMethods con ISIDORE a través del plugin NERD. 


\section{SEPTEMBER 15, $2019 \cdot$ BY ROMBERT STAPEL \\ Analyzing Documents with TF-IDF | Programming Historian}

Introduction by OpenMethods Editor (Rombert Stapel):

The indispensable Programming Historian comes with an introduction to Term Frequency - Inverse Document Frequency (tf-idf) provided by Matthew J. Lavin. The procedure, concerned with specificity of terms in a document, has its origins in information retrieval, but can be applied as an exploratory tool, finding textual similarity, or as a pre-processing tool for machine learning. It is therefore not only useful for textual scholars, but also for historians working with large collections of text.

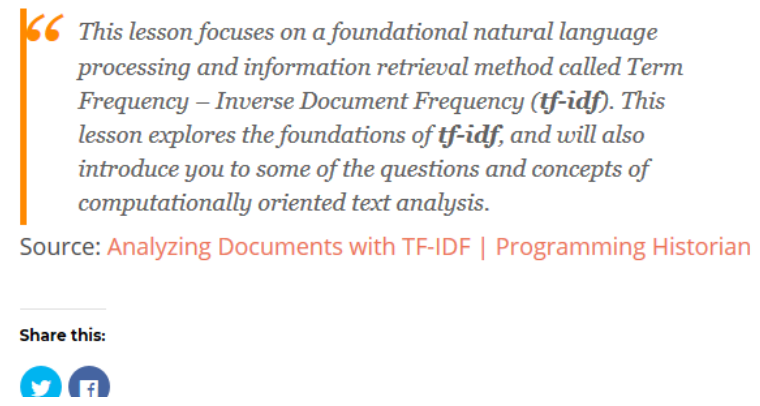

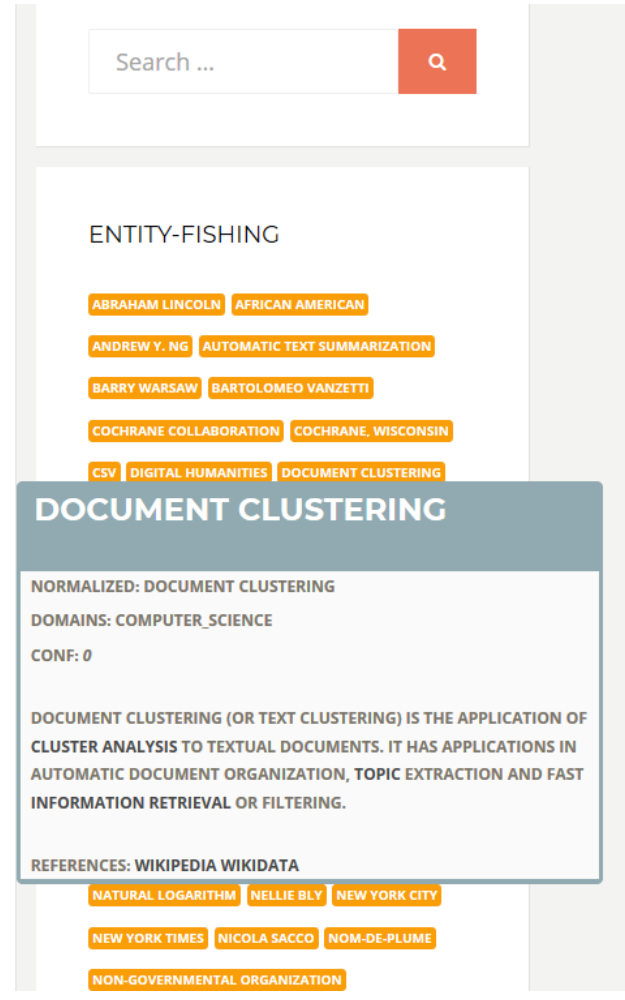

Figura 4. Etiquetadas generadas automáticamente en OpenMethods relacionadas con otras de Wikidata.

abierto. Por un lado, para mejorar el reuso y aumentar la visibilidad de nuestras publicaciones, hemos creado complementos interoperables con otras iniciativas como la plataforma de búsqueda ISIDORE, desarrollada por HumaNum. Se trata del plugin NERD, que reconoce y desambigua las entidades nombradas (ver figura 3). Como una forma de enriquecimiento de contenido, el complemento crea automáticamente etiquetas de las entidades nombradas ofrecidas por NERD cuando se le proporciona el texto completo del artículo original que se ha vuelto a publicar en OpenMethods.

Utilizamos además etiquetas para proponer información adicional proveniente de proyectos basados en Conocimiento Abierto, como Wikipedia y Wikidata. Estas etiquetas son auto-generadas y reusan otras ya utilizadas en Wikidata, qye contribuyen a una mejor capacidad de detección y búsqueda de contenido en OpenMethods y agregan capas de contexto adicionales a nuestro contenido. Este servicio está disponible para contenido en inglés, francés, alemán, italiano y español (ver figura 4).

Por otro lado, para la nominación de contenido reusamos el plugin Pressforward, una iniciativa del campo de las Humanidades Digitales, desarrollada por el Roy Rosenzweig Center for History and New Media at George Mason University, que nos permite crear un flujo de trabajo simple para trabajar con los expertos que se acercan a nuestra comunidad (nominación de contenido, discusión, evaluación, publicación, seguimiento del contenido publicado) ${ }^{9}$. Así mismo, con el fin de enriquecer el contenido abierto preseleccionado y mejorar su búsqueda en el CMS y su visibilidad en la web, las publicaciones se clasifi- can en función de la semántica de la Taxonomía de Actividades Digtales en la Investigación en Humanidades desarrollada por DARIAH, también conocida como TaDiRAH (Borek et al., 2016). TaDiRAH es hoy día un estándar para las Humanidades Digitales, que además, ha sido traducida a diferentes lenguas (por ejemplo, del Rio Riande et al., 2015) (ver figura 5).

Todos los contenidos se acompañan con una breve introducción en inglés, entendido aquí no como lengua de comunicación científica principal, sino, como decíamos más arriba, como la que es compartida por la mayor parte de la comunidad y sirve para glosar el contenido en diferentes lenguas y dar una breve idea acerca de esta a aquellos usuarios que no tiene conocimientos sobre el idioma de las publicaciones.

En síntesis, como decíamos, el proyecto busca ir más allá de las prácticas tradicionales de la comunicación científica desde diversos lugares que apelan a procesos, métodos y tecnologías abiertos (Moranville y Tóth-Czifra, 2018), en una plataforma colaborativa, abierta y en constante crecimiento, ya que recoge las prácticas de la comunidad de humanistas digitales en el mundo (Spiro, 2012). Sus objetivos podrían entonces resumirse en los siguientes enunciados:

1. Dar valor a los métodos no solo a los resultados. OpenMethods está dedicado a la discusión crítica de las herramientas y métodos en Humanidades Digitales. Las herramientas y métodos digitales son productos de investigación genuinos cuyo reconocimiento académico aún está rezagado con respecto a monografías y artículos de revistas especializadas que, lamentablemente, siguen siendo los elementos de valor para 


\section{How does it work?}

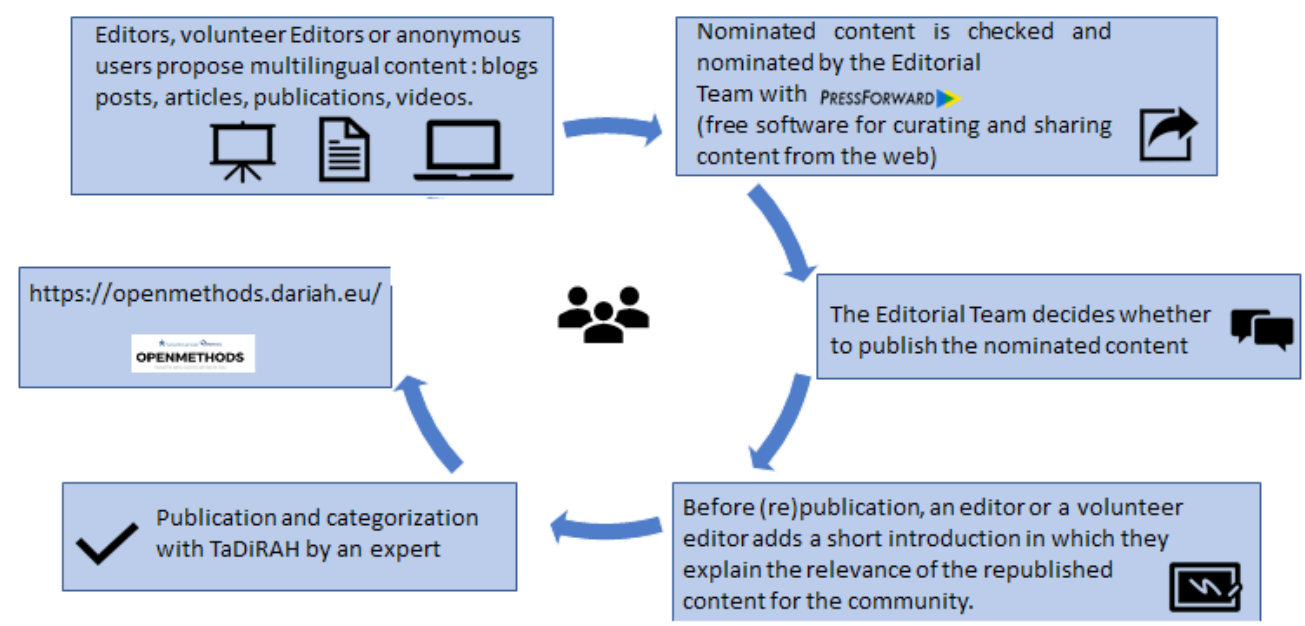

Figura 5. Descripción del flujo de trabajo en la plataforma OpenMethods.

la evaluación académica (Schreibman, Mandell y Olsen, 2011).

2. Ante todo, reusar. Además de difundir el conocimiento y aumentar el reconocimiento entre pares acerca de los recursos digitales existentes en Humanidades Digitales, una misión central del proyecto también es facilitar la cultura de reutilización de estos materiales. Esta aproximación no solo se basa en los contenidos que OpenMethods busca mostrar, sino en el reuso y mejora de desarrollos de las Humanidades Digitales, como Pressfoward, NERD o TaDiRaH, que apela también a la colaboración con centros y proyectos a nivel global (HumaNum, CONICET, Wikimedia).

3. Dar valor a todos los géneros de la publicación científica. OpenMethods incluye una variedad de tipos de contenido republicados que provienen de sitios web personales, blogs, videos, preprints, podcasts, artículos científicos, monografías, y otros géneros como la llamada literatura gris. Poner estos géneros al mismo nivel es otorgarles el valor que se merecen, ya que se están convirtiendo en medios de comunicación cada vez más importantes del trabajo académico (Dacos y Mounier, 2010) que no solo aceleran las discusiones dentro y fuera de la academia, pero también son lo suficientemente flexibles como para dialogar la naturaleza dinámica y multimodal del discurso de los métodos de las Humanidades Digitales.

4. Enriquecer desde lo semántico y lo cultural. Uno de los objetivos clave de la plataforma es ayudar a los investigadores a navegar a través del rico y dinámico paisaje de las Humanidades Digitales con el fin de encontrar las herramientas y métodos más relevantes para su investigación. Como un enriquecimiento de las publicaciones de acceso abierto preseleccionadas, las nominaciones exitosas se clasifican con etiquetas basadas en TaDiRAH y se les agrega una breve introducción en inglés a cada publicación en la que uno de nuestros Editores explica la relevancia del contenido republicado. En este sentido, como expresábamos en líneas precedentes, el inglés deja de ocupar el lugar de lengua única y solo se comprende como la lengua de comunicación científica que la mayoría conoce y utiliza para glosar contenido.

5. Adscribir desde las Humanidades a los principios FAIR (Findability, Accesibility, Interoperability and Reproducibility). Uno de los grandes desafíos del trabajo con datos consiste en facilitar a los humanos y a las máquinas el descubrimiento, acceso, integración y análisis de datos científicos y sus flujos de trabajo. Los principios rectores FAIR (Wilkinson et al., 2016) han sido trabajados principalmente en las ciencias duras, pero aún falta una reflexión propia desde las Humanidades Digitales. OpenMethods adscribe a la necesidad de pensar qué tipos de datos necesitamos para unas Humanidades Digitales más abiertas.

\section{Muchos desafíos y algunas conclusiones}

Nuestro principal objetivo es involucrar a la mayor cantidad de comunidades de humanistas digitales, desde jóvenes investigadores, estudiantes de doctorado, a investigadores en Humanidades Digitales. Esta apertura apunta a reconfigurar el perfil del experto, que se transforma en un representante de métodos particulares en Humanidades Digitales. Evidentemente, para lograr este objetivo y los que hemos mencionado en el apartado anterior, OpenMethods enfrenta varios desafíos dignos de ser mencionados:

1. Alcanzar un número alto de lectores como resultado de una estrategia de divulgación en abierto y a escala global (a través de nuestro canal de Twitter, a través de la mención de otros representantes en proyectos de investigación, de los editores de revistas, o de presentaciones en evento de Humanidades Digitales o Comunicación Científica). No obstante, lenguas, 
diferentes tradiciones disciplinarias, distintos momentos de desarrollo de las Humanidades Digitales y hasta los modos en los que se divide el trabajo en el año lectivo son barreras que muchas veces poco contribuyen a la construcción de unas Humanidades Digitales globales.

2. Encontrar formas de incentivar y dar crédito por el trabajo realizado a los editores a largo plazo es otro problema para nuestro proyecto, ya que el crédito académico sigue pasando principalmente por la publicación científica en revistas indexadas y por el trabajo individual (del Rio Riande, 2018ab).

3. Mejorar la visibilidad y el reconocimiento del trabajo no solo sobre el contenido sino sobre los distintos métodos que hoy sostienen desde las prácticas a las Humanidades Digitales. En algún punto, OpenMethods busca colaborar con la construcción de espacios críticos aún inexistentes en las Humanidades Digitales, como los artículos de datos (Candela et al., 2016).

4. Aportar a la visibilidad y el reconocimiento de culturas diferentes al inglés y, por lo tanto, debilitar la posición hegemónica del idioma inglés dentro del campo de las Humanidades Digitales, incluso si algunos países usan principalmente inglés en sus investigaciones (del Rio Riande et al., 2018ab)10. Como bien han estudiado Vasen y Lujano (2017), existen asimetrías geográficas en la publicación de revistas científicas, sino también hay asimetrías disciplinares e inclusive lingüísticas. Aunque nuestro equipo editorial está totalmente de acuerdo en que el multilingüismo es un valor clave para las Humanidades Digitales y OpenMethods busca reflejar esto en la plataforma, actualmente experimentamos un fuerte sesgo en el conte- nido del idioma inglés, la curación editorial del contenido en idiomas distintos al inglés es considerablemente más lenta (Tóth-Czifra, 2019).

Más allá de estas barreras, creemos que el contenido que propone OpenMethods puede transformarse en una herramienta valiosa y poderosa para fomentar la discusión sobre un área importante pero subrepresentada en las Humanidades Digitales y en la investigación en general: los datos y los métodos, los procesos y no los resultados. Desde su estructura web, el alcance de metablog va más allá de la publicación tradicional, y apunta a una apertura y una inclusión y reuso crítico de literatura gris y de una variedad de tipos de contenido como publicaciones de blog, videos, presentaciones o podcasts. Estos formatos de publicación dinámicos son "recognized and incrementally integrated into more efficient and dynamic workflows of production, improvement, and dissemination of scholarly knowledge in general" (Heller et al., 2014), aunque aún necesitan de un mayor valor, crédito, institucionalización y, a la vez, capacidad de utilización por parte de la comunidad académica. Desde la construcción de comunidad, no solo la diversidad y el multilingüismo están entre sus objetivos, sino que busca redefinir la figura del experto en Humanidades Digitales, que en la colaboración abierta a través de la nominación, selección o edición del contenido a republicar se transforma en experto en tanto representante de métodos particulares en Humanidades Digitales. En síntesis, la apertura propuesta por OpenMethods interroga desde la apertura los paradigmas tradicionales de expertise, colaboración, difusión y publicación. Si la revolución de las Humanidades Digitales todavía está por

\section{JULY 3, 2019 \\ El archivo y la toma de notas. El lugar del software en la interpretación histórica}

Introduction by OpenMethods editor (GImena del Rio): In this article, Nicolás Quiroga reflects on the fundamental place of the note-taking practice in the work of historians. The artcile also reviews some tools for classifying information -which do not substantially affect the note-taking activity - and suggests how the use of these tools can create new digital approaches for historians.

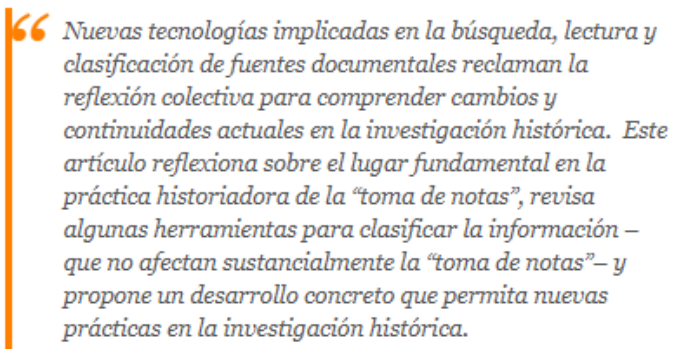

Source: El archivo y la toma de notas. El lugar del software en la interpretación histórica | Quiroga | Revista de Humanidades Digitales

Original date of publication: 28-06.2019

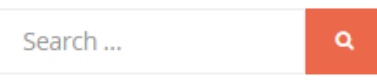

NAMED ENTITY-FISHING

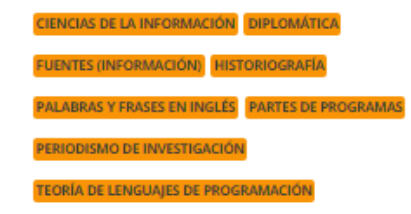

○ ISIDORE SUGGESTIONS

El español internacional en la prensa hispana de los Estados Unidos: hacia una nivelación interdialectal del español en los medios estadounidenses

Análisis del tratamiento de la discapacidad intelectual en los medios online españoles (2010non

Figura 6. Contenido en español en la plataforma OpenMethods. 
Llegar (Clavért, 2013), OpenMetods busca colaborar con ella, haciendo del campo un espacio más equitativo, abierto y global.

\section{Notas al final}

1. Solo por poner un ejemplo, remitimos a la lista de discusión Humanist, donde las intervenciones del tipo What is Digital Humanities? son habituales e incluyen siempre teorías sobre la datación del término: https://dhhumanist.org/volume/33/370/

2. Sobre el problema de la reproducibilidad en la investigación, veáse Allison et al. (2018).

3. La RedHD propuso la herramienta check! Sobre ella puede leerse una descripción en: http://dayofdh2014.matrix.msu.edu/ igalina/2014/04/08/check-formulario-de-evaluacion-para-proyectos-de-humanidades-digitales/. La Sociedad HDH elaboró un extensísimo documento relacionado con la evaluación de los investigadores científicos, que fue luego enviado por dicha asociación a la agencia evaluadora española, la ANECA.

4. Recuperamos aquí también interesantes aproximaciones desde la idea de lo abierto y lo público de la mano de Micah Vandegrift, como las del curso Public Humanities as Scholarly Communication dictado en 2018 en FSCl, Force 11 Scholary Communication Institute: https://medium.com/@mlvandeg/toward-public-humanities-fd3d2183087e.

5. Algunas aproximaciones las trabajamos ya en el Grupo de Interés de la Alliance for the Digital Humanities Organizations (ADHO) llamado Global Outlook (GO::DH). Un resumen con fuerte incidencia en los problemas sobre multiculturalismo y diversidad en las Humanidades Digitales puede leerse en O'Donnell et al. (2016)

6. Véase, por ejemplo, Debat y Babini (2019), acerca del Plan S y América Latina.

7. Si bien, al ser un proyecto eurorpeo, la mayor parte de los editores provienen de países de esa región, resaltamos la dievrsidad de lenguas que aporta cada uno. Además, en el último año, se han incoporado editores de Norte y Sudamérica.

8. En la Figura 2 este hecho puede apreciarse en el segundo de los artículos (Modernes Tool für alte Texte), que aunque escrito en alemán está glosado en inglés.

9. Como parte de nuestro trabajo en la construcción colectiva del conocimiento y del trabajo en abierto, hemos contribuido al código fuente del complemento PressForward, corrigiendo algunos errores y agregando algunas funciones a su herramienta, que se incorporaron en su repositorio principal de Github.

10. Sobre el monopolio lingüístico del inglés en la investigación es vital el trabajo de Priego y Fiormonte (2016) y Albornoz (2017).

\section{Recursos citados}

Clarin. Recuperado de https://www.clarin.eu/

Collections as Data. Recuperado de https://osf.io/m×6uk/wiki/home/

DARIAH. Recuperado de https://www.dariah.eu/

Digital Humanities Data Curation Institute. Recuperado de http:// www.dhcuration.org/institute/

Humanidades Digitales Hispánicas. Recuperado de https://humanidadesdigitaleshispanicas.es

Humanist Discussion Group. Recuperado de https://dhhumanist.org

Laboratorio de Humanidades Digitales del Centro Argentino de Información Científica y Tecnológica del CONICET (HD CAICYT Lab). Recuperado de http://hdlab.space/

New York University. Best Practices in Digital Humanities. Recuperado de https://www.nyu.edu/gsas/dept/fineart/research/digital-humanities-best-practices.htm
Open Context. Recuperado de https://opencontext.org/

Open DH. Recuperado de http://openindh.org/

OpenMethods. Recuperado de https://openmethods.dariah.eu

Open Science Framework (OSF). Recuperado de https://osf.io/sq5a6

Perseus Digital Library. Recuperado de http://www.perseus.tufts. edu/hopper/opensource

Red de Humanidades Digitales. RedHD. Recuperado de http://humanidadesdigitales.net

Stanford University. Best Practices in Digital Humanities. Recuperado de https://digitalhumanities.stanford.edu/digiPre

University of Nebraska. Best Practices in Digital Humanities. Recuperado de https://cdrh.unl.edu/articles/best_practices

\section{Referencias bibliográficas}

Albornoz, D. (2017). The Rise of Big Publishers in Development and What is at Stake. The Knowledge Gap: Geopolitics of Academic Production. Recuperado de http://knowledgegap.org/index. php/2017/09/20/the-rise-of-big-publishers-in-development-whatis-at-stake/

Allison, D. B., Shiffrin, R. M., y Stodden, V. (2018). Reproducibility of Research: Issues and Proposed Remedies. Proceedings of the National Academy of Sciences of the United States of America, PNAS. https://doi.org/10.1073/pnas.1802324115

Alperin, J. P., y Fischman, G. (2015). Hecho en Latinoamérica: acceso abierto, revistas académicas e innovaciones regionales. Ciudad Autónoma de Buenos Aires: CLACSO. Recuperado de: http://biblioteca. clacso.edu.ar/clacso/se/20150722110704/HechoEnLatinoamerica.pdf

Anderson, S., y McPherson, T. (2011). Engaging Digital Scholarship: Thoughts on Evaluating Multimedia Scholarship. Profession16, 136-151.

Appel, A., Lujano Vilchis, I., y Albagli, S. (2018). Open Science Practices Adopted by Latin American Caribbean Open Access Journals. ELPUB2018, Toronto, Canada. Recuperado de: https://hal.archives-ouvertes.fr/hal-01800164v3

Borek, L., Dombrowski, Q.., Perkins, J., y Schöch, C. (2016). TaDiRAH: a Case Study in Pragmatic Classification. Digital Humanities Quarterly, 10(1). Recuperado de http://www.digitalhumanities.org/dhq/ vol/10/1/000235/000235.html

Candela, L., Castelli, D., Manghi, P., y Tani, A. (2015). Data Journals: A Survey. Journal of the Association for Information Science and Technology, 66(9),1747-1762. https://doi.org/10.1002/asi.23358

Clavért, F. (2013). The Digital Humanities Multicultural Revolution Did not Happen Yet. Recuperado de: https://histnum.hypotheses. org/1546\#comments

Dacos, M., y Mounier, P. (2010). Les carnets de recherche en ligne, espace d'une conversation scientifique décentrée. Paris: Albin Michel.

Debat, H y Babini, D. (2019). Plan S in Latin America: A Precautionary Note. PeerJ Preprints. https://doi.org/10.7287/peerj.preprints.27834v2

del Rio Riande, G. (2015). Humanidades Digitales. Mito, actualidad y condiciones de posibilidad en España y América Latina. ArtyHum, monográfico 1, 7-19. Recuperado de: https://www.artyhum.com/ descargas/monograficos/MONOGR\%C3\%81FICO\%20HD.pdf

del Rio Riande, G. (2016). De todo lo visible y lo invisible o volver a 
pensar la investigación en Humanidades Digitales. Signa. Revista de la Asociación Española de Semiótica, (25), 95-108.

del Rio Riande, G. (2018a). Defining Collaboration in the (Global) Digital Humanities. FORCE2019, Mc. Gill University, Montreal, Canadá Recuperado de: https://zenodo.org/record/1470688\#.XdZk4y0-8UE

del Rio Riande, G. (2018b). Humanidades Digitales bajo la lupa: Investigación abierta y evaluación científica. Ex Libris, 7. Recuperado de: http://revistas.filo.uba.ar/index.php/exlibris/article/view/3177

del Rio Riande, G., De León, R., y Ferreyra, D. (2015). Taxonomía de Investigación Digital en Humanidades, TaDIRAH de DARIAH. Recuperado de: http://www.vocabularyserver.com/tadirah/es/

del Rio Riande, G. del, Brussa, V., Hathcock, A. (2018). Dónde está lo abierto en las Humanidades Digitales?. III Congreso Internacional de la Asociación de Humanidades Digitales (AAHD). La cultura de los datos. Recuperado de: http://hdl.handle.net/2133/13458

Engelhardt, C., Leone, C., Larrousse, N., Montulio, D., y Moranville, Y. (2017). Open Humanities Methods Review Journal. [Research Report] DARIAH; TGIR HumaNum (UMS 3598); Göttingen State University Library. Recuperado de https://hal.archives-ouvertes.fr/ hal-01685852

Fiormonte, D. (2012). Towards and Cultural Critique of the Digital Humanities. In: Thaller, M. (ed.) Controversies around the Digital Humanities, 59-76.

Fiormonte, D. (2014). Digital Humanities from a Global Perspective. Laboratorio dell'ISPF XI. https://doi.org/10.12862/ispf14L203

Fiormonte, D., del Rio Riande, G. (En prensa). Dalla periferia dell'impero. Digital Humanities e diversità culturale. En: Ciotti, F. y Tomasi, F. (erd.) Digital Humanities: metodi, strumenti, saperi.

Fitzpatrick, K. (2010). Reporting from the Digital Humanities 2010 Conference. The Chronichle of Higher Education. Recuperado de: https://www.chronicle.com/blogs/profhacker/reporting-from-the-digital-humanities-2010-conference/25473

FOSTER (s/d). Open Science Definition. Recuperado de: https://www. fosteropenscience.eu/foster-taxonomy/open-science-definition

Galina, I. (2014). Geographical and Linguistic Diversity in the Digital Humanties. Literary and Linguistic Computing, 29(3), 307-316.

Heller, L., The, R., y Bartling, S. (2014). Dynamic Publication Formats and Collaborative Authoring. In: Bartling, S., y Friesike, S. (Eds.). Opening Science. Cham: Springer International Publishing, 191-211. Recuperado de: https://link.springer.com/chapter/10.100 7\%2F978-3-319-00026-8_13

Hess, Ch., y Ostrom, E. (Eds.) (2006). Understanding Knowledge as a Commons. From Theory to Practice. Cambridge, Massachusetts: MIT Press.

Jones, S. (2016). Roberto Busa, S.J., and the Emergence of Humanities Computing: The Priest and the Punched Cards. New York: Routledge.

Kirschenbaum, M. (2010). What is Digital Humanities and What is Doing in English Departments?. ADE Bulletin, (150), 1-7. Recuperado de: https://mkirschenbaum.files.wordpress.com/2011/01/kirschenbaum_ade150.pdf

Larsen, P., y von Ins, M. (2010). The Rate of Growth in Scientific Publication and the Decline in Coverage Provided by Science Citation Index. Scientometrics, 84(3), 575-603.

Moretti, F. (2005). Distant Reading. London, New York: Verso.

Moranville, Y., y Tóth-Czifra, E. (2018). Leveraging on the power of expert content curation: the OpenMethods metablog. EADH 2018: Data in Digital Humanities, EADH, Dec 2018, Galway, Iceland. Recuperado de: https://halshs.archives-ouvertes.fr/halshs-02010915/ document

O'Donnell D. P., Bordalejo, B., Murray Ray, P., del Rio Riande, G., y González-Blanco, E. (2016). Boundary Land: Diversity as a defining feature of the Digital Humanities. Jagiellonian University \& Pedagogical University (Krákow). Recuperado de: http://e-spacio.uned.es/ fez/eserv/bibliuned:363-Egonzalez5/Gonzalez_Blanco_Elena_BoundaryDH.pdf

Sahle, P. (2013). DH studieren! Auf dem Weg zu einem Kern- und Referenzcurriculum der Digital Humanities. DARIAH-DE Working Papers Nr. 1. Göttingen: GOEDOC 2013.

Schallier, W., del Rio Riande, G., Hathcock, A., y O'Donnell, D. (2018). Open South: The Open Science Experience in Latin America and the Caribbean. Course at FORCE11 Scholarly Communication Institute (FSCI), UCSD, La Jolla, San Diego. Recuperado de: https://docs.google. com/document/d/1qo48xSEvf6l6Q_K6GkCTSxxp-nfv_DNe2MMWuSJMI8PA/edit\#heading=h.iebqidq4r2v7

Schreibman, S., Siemens, R., y Unsworth, J. (2004). A Companion to Digital Humanities. Oxford: Blackwell.

Schreibman, S., Mandell, L., y Olsen, S. (2011). Evaluating Digital Scholarchip. Introduction. Profession, (2011), 123-135. https://doi. org/10.1632/prof.2011.2011.1.123

Spiro, L. (2012). This Is Why We Fight: Defining the Values of the Digital Humanities. In: Gold, M. K. (Ed.). Debates in the Digital Humanities (pp. 16-35). Minnesota: University of Minnesota Press.

Suber, P. (2006). Open Access Overview. Focusing on Open Access to Peer-Reviewed Research Articles and their Preprints. Recuperado de: http://legacy.earlham.edu/ peters/fos/overview.htm

Tennant, J. (2016). Breaking the Traditional Mould of Peer Review: Why We Need a More Transparent Process of Research Evaluation. LSE Impact blog. [Blog]. Recuperado de: https://blogs.lse.ac.uk/ impactofsocialsciences/2016/02/17/breaking-the-traditional-mouldof-peer-review/

Svensson, A. (2014). El término Humanidades Digitales y los Estudios Latinoamericanos: una revisión bibliográfica. Anuario Americanista Europeo, 2221-3872, 12, 1-28. Recuperado de: http://www.red-redial. net/revista/anuario-americanista-europeo/article/view/281/274

Tóth-Czifra, E. (2019). 6 innovations from the humanities that make Open Access publishing a reality to everyone. DARIAH Open. Open Scholarly Practices in the Arts and the Humanities. Recuperado de: https://dariahopen.hypotheses.org/529

Vanhoutte, E. (2013). The Gates of Hell: History and Definition of Digital | Humanities | Computing. In: Terras, M., Nyhan, J. y Vanhoutte, E. (Eds.) Defining Digital Humanities, (p. 119-156). Franham: Ashgate Publishing.

Vasen, F., y Lujano Vilchis, I. (2017). Sistemas nacionales de clasificación de revistas científicas en América Latina: tendencias recientes e implicaciones para la evaluación académica en ciencias sociales. Revista mexicana de ciencias políticas y sociales, 62(231), 199-228. Recuperado de: http://www.scielo.org.mx/scielo.php?script=sci_ arttext\&pid=S0185-19182017000300199\&lng=es\&tlng=es

Wilkinson, M.D, Dumontier, M. et al. (2016). The FAIR Guiding Principles for scientific Data Management and Stewardship. Scientific Data, 3, Article number: 160018. Recuperado de: https://doi.org/10.1038/ sdata.2016.18 
Wouters, P., Beaulieu, A., Scharnhorst, A., y Wyatt, S. (Eds.) (2013). Virtual knowledge: Experimenting in the Humanities and the Social Sciences. Cambridge, MA: The MIT Press.

\section{CV}

Gimena del Rio Riande. Es Doctora en Filología Románica y Magister en Estudios Literarios por la Universidad Complutense de Madrid, y es Experta en Investigación y Recuperación del Patrimonio Literario por la Universidad Autónoma de Madrid. Licenciada y Profesora en Letras por la Universidad de Buenos Aires, es en la actualidad Investigadora Adjunta en el Instituto de Investigaciones Bibliográficas y Crítica Textual (IIBICRIT), CONICET (Argentina). Dirige HD CAICYT Lab (CONICET), el laboratorio de Humanidades Digitales del CAICYT y co-dirige el Máster de Humanidades Digitales de la UNED (Madrid, España). Sus líneas de investigación se recortan en la
Humanidades Digitales, la edición digital académica en todos sus formatos y la Investigación Abierta. Sitio web: https:// www.aacademica.org/gimena.delrio.riande

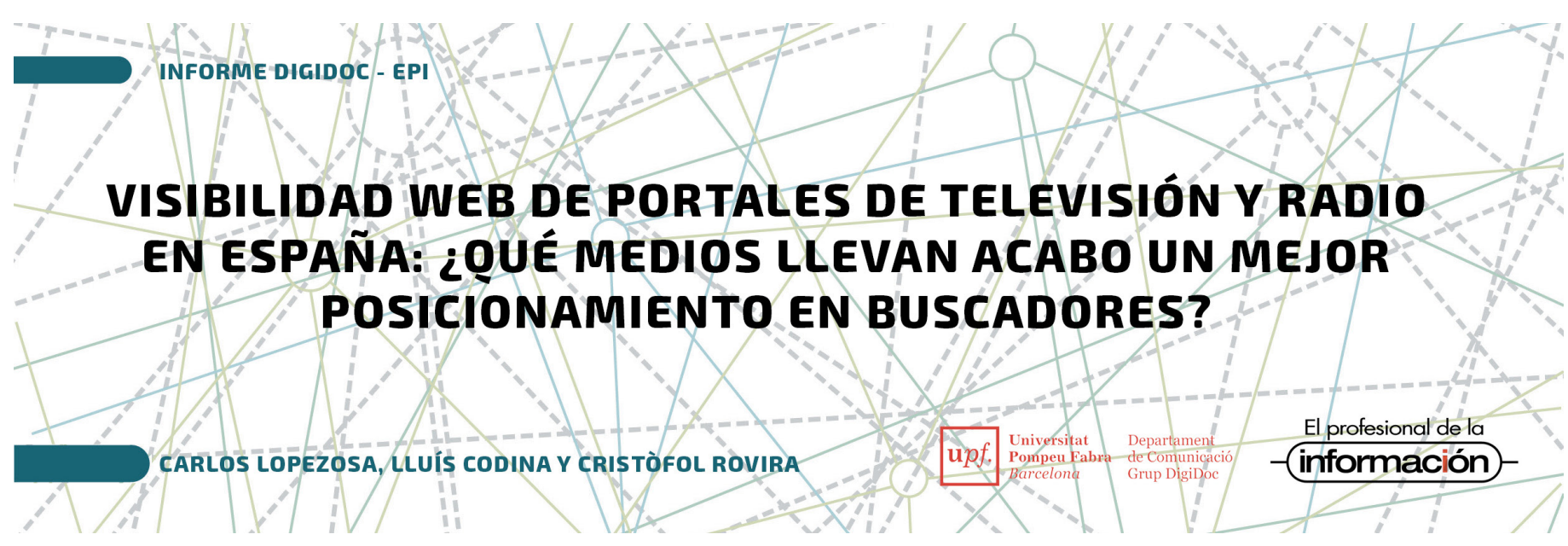

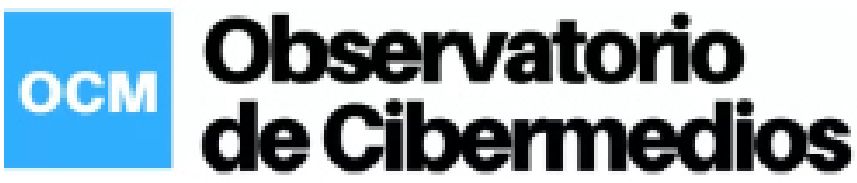

https://observatoriocibermedios.upf.edu/

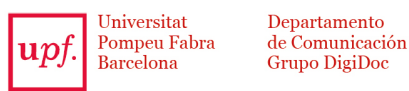

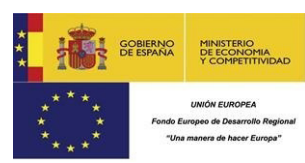

El Observatorio de Cibermedios (OCM) forma parte del proyecto del Plan Nacional "Creación y contenido interactivo en la comunicación de información audiovisual: audiencias, diseño, sistemas y formatos". CSO2015-64955-C4-2-R (MINECO/ FEDER), Ministerio de Economía y Competitividad (España). 